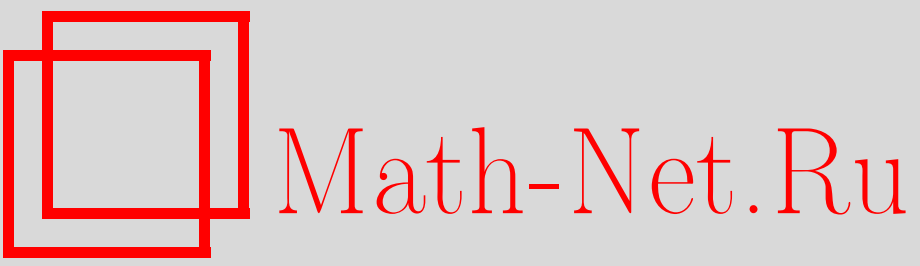

И. Т. Хабибуллин, Уравнение КдФ на полуоси с нулевым краевым условием, TMФ, 1999, том 119, номер 3, 397-404

DOI: https://doi.org/10.4213/tmf747

Использование Общероссийского математического портала Math-Net.Ru подразумевает, что вы прочитали и согласны с пользовательским соглашением

http://www.mathnet.ru/rus/agreement

Параметры загрузки:

IP : 54.157 .27 .8

26 апреля 2023 г., 13:34:59 


\author{
ТЕОРЕТИЧЕСКАЯ \\ И МАТЕМАТИЧЕСКАЯ \\ ФИЗИКА \\ Том 119, № 3 \\ июнь, 1999
}

(C) 1999 г.

И.Т. Хабибуллин*

\title{
УРАВНЕНИЕ КДФ НА ПОЛУОСИ С НУЛЕВЫМ КРАЕВЫМ УСЛОВИЕМ
}

Начально-краевая задача с краевым условием $\left.u\right|_{x=0}=0,\left.u_{x x}\right|_{x=0}=0$ для уравнения $\mathrm{Kд} \Phi$ интегрируется методом обратной задачи рассеяния. Временная динамика матрицы рассеяния эффективно определяется из условия согласования спектров двух дифференциальных операторов, задающих $L-A$-пару.

\section{1. ВВЕДЕНИЕ}

Метод обратной задачи рассеяния (МОЗР) позволяет достаточно полно исследовать задачу Коши для нелинейных интегрируемых уравнений [1]. В случае одной пространственной переменной решение задачи Коши сводится к последовательному решению двух линейных задач: построению фундаментального решения линейной системы обыкновенных дифференциальных уравнений и решению матричной задачи Римана об аналитическом сопряжении функций на комплексной плоскости, равносильной системе сингулярных интегральных уравнений. Многие прикладные задачи формулируются в виде начально-краевых задач для нелинейных дифференциальных уравнений в частных производных. Начально-краевые задачи для уравнений типа уравнения Кортевега-де $\Phi$ риза (КдФ) и нелинейного уравнения Шредингера (НУШ) несомненно представляют прикладной интерес (см., например, $[2-5])$ и поэтому являются предметом активного изучения уже в течение ряда лет. После серии работ Итса и Фокаса [6-8] стало ясно, что при произвольных краевых условиях начально-краевую задачу для упомянутых выше уравнений не удается решить столь же эффективно, как задачу Коши на всей оси. Однако есть некоторый выделенный класс граничных условий, полностью совместимых со свойством интегрируемости, для которых начально-краевая задача эффективно вкладывается в схему МОЗР. Различные примеры таких краевых условий обсуждались в работах $[6,9-12]$. Симметрийньй тест, разработанный в $[13,14]$, позволяет полностью описать классы интегрируемых краевых условий для интегрируемых уравнений в размерности $1+1$. Например [15], уравнение КдФ совместимо с краевыми условиями типа $\left.u(x, t)\right|_{x=0}=a,\left.u_{x x}(x, t)\right|_{x=0}=b$, где $a$ и $b-$ произвольные константы. Регулярные и

\footnotetext{
* Институт математики Уфимского научного центра РАН, Уфа, Россия. E-mail: ihabib@imat.rb.ru
} 
вешественные алгебро-геометрические решения уравнения КдФ, удовлетворяющие таким краевым условиям, описаны в статье [16]. Класс интегрируемых краевых условий сравнительно узок, однако они могут служить основой для изучения влияния общего краевого условия, например, методами теории возмушений.

В настояшей работе исследуется начально-краевая задача на полуоси для уравнения КдФ с произвольными начальными условиями в наиболее простом случае, когда $a=$ $b=0$ :

$$
\begin{aligned}
u_{t} & =u_{x x x}-6 u u_{x}, \quad x>0, \quad t>0, \\
\left.u\right|_{x=0} & =0,\left.\quad u_{x x}\right|_{x=0}=0, \\
\left.u\right|_{t=0} & =u_{0}(x),\left.\quad u_{0}(x)\right|_{x \rightarrow+\infty} \rightarrow 0 .
\end{aligned}
$$

На начальную функцию $u_{0}(x)$ накладываются условия гладкости и быстрого убывания, а также условия согласования с краевым условием $(2)$ в угловой точке: $u_{0}(0)=$ $u_{0 x x}(0)=0$. Ниже мы покажем, что при выполнении этих условий задача (1)-(3) эффективно вкладывается в схему МОЗР. Заметим, что задача (1)-(3) является корректно поставленной. Вопросы сушествования и единственности для нее хорошо исследованы (см., например, [17]).

Как известно [1], уравнение КдФ является условием совместности следующих двух систем линейных уравнений по $x$ и по $t$ :

$$
\begin{aligned}
& Y_{x}=U Y, \\
& Y_{t}=V Y,
\end{aligned}
$$

где матрицы коэффициентов имеют вид

$$
U=\left(\begin{array}{cc}
0 & 1 \\
u-\lambda & 0
\end{array}\right), \quad V=\left(\begin{array}{cc}
u_{x} & -4 \lambda-2 u \\
u_{x x}-(4 \lambda+2 u)(u-\lambda) & -u_{x}
\end{array}\right) .
$$

Легко проверить, что краевые условия (2) выполнены тогда и только тогда, когда уравнение (5) вдоль прямой $x=0$ принимает вид

$$
Y_{t}=\left(\begin{array}{cc}
u_{x}(0, t) & -4 \lambda \\
4 \lambda^{2} & -u_{x}(0, t)
\end{array}\right) Y .
$$

Иначе говоря, линейные системы уравнений (4)-(6) задают представление Лакса начально-краевой задачи (1)-(3).

Основное отличие начально-краевой задачи для интегрируемых уравнений от задачи Коши для тех же уравнений состоит в том, что здесь матрица рассеяния неявным образом зависит от времени $t$. Для описания временной динамики матрицы рассеяния приходится привлекать уравнение (6) и сначала условно, так как коэффициент $u_{x}(0, t)$ также неизвестен, решать для этой системы "задачу рассеяния". Конкретное значение собственной функции вдоль граничной полупрямой $x=0, t>0$ мы ишем из условия согласования задач рассеяния по $x$ и по $t$ в угловой точке $x=0, t=0$. Наше наблюдение состоит в том, что для интегрируемых краевых условий условие согласования позволяет эффективно определить собственную функцию вдоль границы $x=0, t>0$. 


\section{2. ПРЯМАЯ ЗАДАЧА РАССЕЯНИЯ НА ПОЛУОСИ}

Напомним, что в основе МОЗР лежит нелинейная нелокальная замена переменной, линеаризующая исходное нелинейное уравнение (1). Замена переменной определяется как преобразование, переводящее коэффициенты системы уравнений (4) в некоторые "спектральные" данные, выражаюшиеся через фундаментальное решение системы. Следуя этому принщипу, построим матричные решения системы уравнений (4), предписывая им при всех действительных $\xi=\sqrt{\lambda}$ следующее асимптотическое поведение:

$$
\begin{array}{ll}
Y_{1}(x, t, \xi) \rightarrow T(\xi) e^{i x \xi \sigma_{3}}, & x \rightarrow+\infty, \\
Y_{2}(x, t, \xi) \rightarrow T(\xi), & x \rightarrow 0,
\end{array}
$$

где

$$
\sigma_{3}=\left(\begin{array}{cc}
1 & 0 \\
0 & -1
\end{array}\right), \quad T(\xi)=\left(\begin{array}{cc}
1 & 1 \\
i \xi & -i \xi
\end{array}\right) .
$$

Как известно [1], векторы-столбцы $\psi_{k}(x, t, \xi)$ и $\phi_{k}(x, t, \xi), k=1,2$, матриц $Y_{1}=\left(\psi_{1}, \phi_{1}\right)$ и $Y_{2}=\left(\phi_{2}, \psi_{2}\right)$ допускают аналитическое продолжение по параметру $\xi$ с вешественной оси в комплексную плоскость. Более того, $Y_{2}(x, t, \xi)$ является целой аналитической функцией, а ее столбцы имеют в соответствуюших полуплоскостях следующее асимптотическое поведение при $\xi \rightarrow \infty$ :

$$
\begin{array}{ll}
\phi_{2}(x, t, \xi) \rightarrow e^{i x \xi}\left(\begin{array}{c}
1 \\
i \xi
\end{array}\right)\left(1+O\left(\xi^{-1}\right)\right), & \operatorname{Im} \xi \leqslant 0, \\
\psi_{2}(x, t, \xi) \rightarrow e^{-i x \xi}\left(\begin{array}{c}
1 \\
-i \xi
\end{array}\right)\left(1+O\left(\xi^{-1}\right)\right), & \operatorname{Im} \xi \geqslant 0 .
\end{array}
$$

Формулы (9), (10) проще всего доказываются сведением к равносильным интегральным уравнениям. Например, как нетрудно проверить, первая компонента вектора $\psi_{2}(x, t, \xi)$ является решением интегрального уравнения вида

$$
y(x, \xi)+\int_{0}^{x} u(s) \frac{\sin \xi(x-s)}{\xi} y(s, \xi) d s=e^{-i \xi x} .
$$

Столбцы же другого матричного решения $Y_{1}$ продолжаются, вообше говоря, лиш в полуплоскости $\operatorname{Im} \xi<0$ и $\operatorname{Im} \xi>0$, соответственно, где имеют аналогичные асимптотики при $\xi \rightarrow \infty$ :

$$
\begin{array}{ll}
\phi_{1}(x, t, \xi) \rightarrow e^{-i x \xi}\left(\begin{array}{c}
1 \\
-i \xi
\end{array}\right)\left(1+O\left(\xi^{-1}\right)\right), & \operatorname{Im} \xi \leqslant 0, \\
\psi_{1}(x, t, \xi) \rightarrow e^{i x \xi}\left(\begin{array}{c}
1 \\
i \xi
\end{array}\right)\left(1+O\left(\xi^{-1}\right)\right), & \operatorname{Im} \xi \geqslant 0 .
\end{array}
$$

Матрицу рассеяния системы уравнений (4) на полуоси определим как отношение двух фундаментальных решений: $s(\xi)=Y_{2}^{-1} Y_{1}$. Сравним эту матрицу рассеяния с матрицей рассеяния задачи на всей оси $-\infty<x<+\infty$ с быстроубываюшим потеншиалом (см., 
например, [18]). Можно показать, что если в задаче на всей оси потенциал $u(x)$ равен нулю при всех $x \leqslant 0$, то ее стандартная матрица рассеяния в точности совпадает с матрицей рассеяния на полуоси, введеной выше. Это обстоятельство упрошает проблему описания области значений отображения, переводящего $u(x)$ в $s(\xi)$. Искомая область значений является подклассом во множестве всех матриц рассеяния, соответствующих классу убывающих потенциалов, заданных на всей оси. Подкласс характеризуется тем, что наряду с диагональными элементами $s_{11}(\xi)$ и $s_{22}(\xi)$ внедиагональные элементы $s_{21}(\xi)$ и $s_{12}(\xi)$ матрищы $s(\xi)$ также допускают аналитическое продолжение в области $\operatorname{Im} \xi>0$ и $\operatorname{Im} \xi<0$, соответственно.

Взяв векторы $\phi_{k}$ и $\psi_{k}$ в качестве столбцов, составим матрищы $\phi$ и $\psi$ по следующему правилу:

$$
\begin{aligned}
\phi(x, \xi) & =\left(\phi_{2}, \phi_{1} s_{22}^{-1}\right)(x, \xi) e^{-i x \xi \sigma_{3}} \\
\psi(x, \xi) & =\left(\psi_{1} s_{11}^{-1}, \psi_{2}\right)(x, \xi) e^{-i x \xi \sigma_{3}}
\end{aligned}
$$

По построению функции $\phi(x, \xi)$ и $\psi(x, \xi)$ аналитичны соответственно в нижней и верхней полуплоскостях, поэтому эти две функции совместно можно рассматривать как одну кусочно-аналитическую функцию, определенную на всей комплексной плоскости и совершаюшую скачок при переходе через вешественную ось $\operatorname{Im} \xi=0$ :

$$
\phi(x, \xi)=\psi(x, \xi) r(x, \xi)
$$

Матрица сопряжения $r(x, \xi)$, задаюшая скачок, достаточно просто зависит от $x, x \geqslant 0$ :

$$
r(x, \xi)=e^{i x \xi \sigma_{3}} r(0, \xi) e^{-i x \xi \sigma_{3}} .
$$

Из асимптотических представлений, приведенных выше, следует, что

$$
\begin{array}{llll}
\phi(x, \xi) \rightarrow T(\xi) & \text { при } & \operatorname{Im} \xi \leqslant 0, & \xi \rightarrow \infty \\
\psi(x, \xi) \rightarrow T(\xi) & \text { при } & \operatorname{Im} \xi \geqslant 0, & \xi \rightarrow \infty
\end{array}
$$

Поскольку функции $s_{11}(\xi)$ и $s_{22}(\xi)$ имеют в случае обшего положения полюс в точке $\xi=0$, то из формул (13), (14) вытекает

$$
\phi(x, 0)\left(\begin{array}{l}
0 \\
1
\end{array}\right)=0, \quad \psi(x, 0)\left(\begin{array}{l}
1 \\
0
\end{array}\right)=0
$$

Особый случай, когда функция $s(\xi)$ в нуле ограничена, исследуется аналогично. При этом следует иметь в виду, что матришы $\phi(x, \xi)$ и $\psi(x, \xi)$ становятся невырожденными в нуле и условие (19) перестает выполняться.

Матрища сопряжения явно выражается в терминах элементов матрицы рассеяния:

$$
r(x, \xi)=\left(\begin{array}{cc}
1 & s_{12} s_{22}^{-1} e^{2 i x \xi} \\
-s_{21} s_{11}^{-1} e^{-2 i x \xi} & s_{11}^{-1} s_{22}^{-1}
\end{array}\right) .
$$


Решением задачи рассеяния на полуоси $x>0$ в фиксированной точке $\xi$ назовем невырожденное матричное решение $Y(x, \xi)$ системы уравнений (4) такое, что функция $Y(x, \xi) e^{-i x \xi \sigma_{3}}$ ограничена при всех $x \geqslant 0$. Такая формулировка задачи рассеяния (для задачи на всей оси) была дана ранее в работе Шабата [19]. Ясно, что функции $Y(x, \xi)=$ $\phi(x, \xi) e^{i x \xi \sigma_{3}}$ и $Y(x, \xi)=\psi(x, \xi) e^{i x \xi \sigma_{3}}$ доставляют некоторое решение задачи рассеяния определенное всюду на комплексной плоскости, за исключением точек $\xi$, где $s_{22}(\xi)=0$, если $\operatorname{Im} \xi<0$, и $s_{11}(\xi)=0$, если $\operatorname{Im} \xi>0$. Заметим, что решение этой задачи, в отличие от аналогичной задачи на всей прямой, содержит некоторый произвол. Например, при $\operatorname{Im} \xi<0$ решение задачи рассеяния можно умножать на невырож денную нижнетреугольную матрицу, а при $\operatorname{Im} \xi>0$ - на невырожденную верхнетреугольную. Ниже нам понадобятся явные представления матриц $\phi$ и $\psi$ через элементы матриц рассеяния

$$
\begin{aligned}
T^{-1}(\xi) \phi(0, \xi) & =\left(\begin{array}{ccc}
1 & s_{12}(\xi) s_{22}^{-1}(\xi) \\
0 & 1
\end{array}\right), \\
T^{-1}(\xi) \psi(0, \xi) & =\left(\begin{array}{cc}
1 & 0 \\
s_{21}(\xi) s_{11}^{-1}(\xi) & 1
\end{array}\right) .
\end{aligned}
$$

Следует подчеркнуть, что матрица рассеяния задачи на полуоси неявным образом зависит от времени $t$ и в этом основная трудность, возникающая при переходе от задачи Коши к начально-краевой задаче. Нетрудно показать, пользуясь уравнением (6), что зависимость матрицы рассеяния от времени определяется следующей системой дифференциальных уравнений:

$$
s_{t}=4 i \xi^{3}\left[s, \sigma_{3}\right]+u_{x}(0, t) \sigma_{1} s
$$

где $\sigma_{1}=\left(\begin{array}{ll}0 & 1 \\ 1 & 0\end{array}\right)$ - матрица Паули. Система уравнений (23) является недоопределенной, поскольку функция $u_{x}(0, t)$, входящая в коэффициенты системы уравнений $(23)$, является неизвестной. Однако дополнительное требование - сохранение свойства аналитичности столбцов матрищы $s(\xi, t)$ в полуплоскостях $\operatorname{Im} \xi>0, \operatorname{Im} \xi<0$ при всех $t>0-$ в конечном счете позволяет определить оба неизвестных объекта $u_{x}(0, t)$ и $s(\xi, t)$.

В следуюшем разделе мы сведем задачу отыскания матрицы $s(\xi, t)$ к некоторой задаче Римана.

\section{3. ВРЕМЕННАЯ ДИНАМИКА МАТРИЦЫ РАССЕЯНИЯ}

Исследуем уравнение (6), описывающее временную динамику собственной функции в граничной точке $x=0$. При помощи линейной замены переменных $Y=T \widetilde{Y}$ приведем эту систему уравнений к виду

$$
\tilde{Y}_{t}=\left(-4 i \xi^{3} \sigma_{3}+u_{x}(0, t) \sigma_{1}\right) \tilde{Y} .
$$

Полагая в $(24) \xi^{3}=\mu$, придем к системе уравнений Захарова-Шабата, для которой задача рассеяния хорошо изучена в связи с ее приложениями в теории интегрируемости [1]. Предположим, считая $u_{x}(0, t)$ гладкой функцией, что системе уравнений $(24)$ соответствует некоторая задача Римана со скачком вдоль прямой $\operatorname{Im} \mu=0$ :

$$
\Phi(t, \mu)=\Psi(t, \mu) R(t, \mu) .
$$


Здесь функции $\Phi$ и $\Psi$ аналитичны соответственно в полуплоскостях $\operatorname{Im} \mu<0$ и $\operatorname{Im} \mu>0$ и нормированы условием на бесконечности $\Phi(t, \infty)=\Psi(t, \infty)=1$. При этом функции $\Phi(t, \mu) e^{-4 i \mu t \sigma_{3}}, \Psi(t, \mu) e^{-4 i \mu t \sigma_{3}}$ являются решениями системы уравнений $(24)$, и поэтому матрица сопряжения удовлетворяет условию

$$
R(t, \mu)=e^{-4 i \mu t \sigma_{3}} R(0, \mu) e^{4 i \mu t \sigma_{3}} .
$$

Факторизация (25) рассматривается лишь для положительных значений времени $t$, поэтому в выборе конкретного решения “задачи рассеяния по $t$ ”, т.е. в выборе функций $\Psi(t, \mu)$ и $\Phi(t, \mu)$, имеется произвол в умножении на нижнетреугольную и верхнетреугольную матрицы, соответственно. Поэтому без ограничения обшности можно этим функциям предписать следуюшие треугольные матричные структуры при $t=0$ :

$$
\Psi(0, \mu)=\left(\begin{array}{ll}
1 & * \\
0 & 1
\end{array}\right), \quad \Phi(0, \mu)=\left(\begin{array}{ll}
1 & 0 \\
* & 1
\end{array}\right)
$$

Ясно, что контур сопряжения $\operatorname{Im} \mu=\operatorname{Im} \xi^{3}=0$ в задаче Римана (25) распадается в плоскости $\xi$ на систему лучей $\left\{l_{j}\right\}_{j=1}^{j=6}$, исходящих из начала координат с углами наклона к положительному направлению оси $\operatorname{Im} \xi=0$, равными $\alpha_{j}=\frac{\pi}{3}(j-1)$. Обозначим через $I_{j}$ внутренность угла, образованного лучами $l_{j+1}$ и $l_{j}$ (см. рисунок).

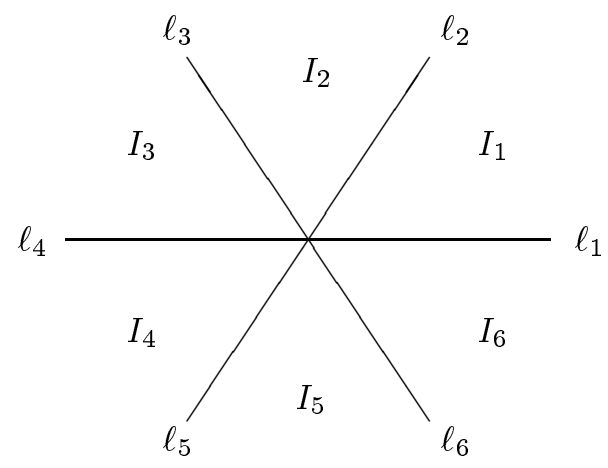

Контур сопряжения.

Воспользуемся совместностью систем уравнений (4) и (5) в четверти плоскости $x \geqslant 0$, $t \geqslant 0$. Совместность означает, что в точке $x=0, t=0$ функции $T \Phi(t, \mu), T \Psi(t, \mu)$ и $\phi(x, \xi), \psi(x, \xi)$ должны быть согласованы для всех значений спектрального параметра $\xi$. Условия согласования решений задачи рассеяния в угловой точке понимаются как условия существования треугольных матричных функций $\delta, \tilde{\delta}$ таких, что выполнены следуюшие равенства в секторах $I_{j}$ :

$$
\begin{aligned}
& \psi(0, \xi) \delta_{u 1}=T(\xi) \Psi(0, \mu) \tilde{\delta}_{l 1}, \quad \xi \in I_{1} \\
& \psi(0, \xi) \delta_{u 2}=T(\xi) \Phi(0, \mu) \tilde{\delta}_{u 2}, \quad \xi \in I_{2}
\end{aligned}
$$




$$
\begin{aligned}
\psi(0, \xi) \delta_{u 3} & =T(\xi) \Psi(0, \mu) \tilde{\delta}_{l 3}, \quad \xi \in I_{3}, \\
\phi(0, \xi) \delta_{l 4} & =T(\xi) \Phi(0, \mu) \tilde{\delta}_{u 4}, \quad \xi \in I_{4} \\
\phi(0, \xi) \delta_{l 5} & =T(\xi) \Psi(0, \mu) \tilde{\delta}_{l 5}, \quad \xi \in I_{5} \\
\phi(0, \xi) \delta_{l 6} & =T(\xi) \Phi(0, \mu) \tilde{\delta}_{u 6}, \quad \xi \in I_{6} .
\end{aligned}
$$

Здесь индексы $l$ (lower) и $u$ (upper) указывают на нижнюю или верхнюю треугольность матрицы, числовой индекс обозначает номер сектора, в котором изменяется параметр $\xi$. Заметим, что в системе уравнений (28)-(33) неизвестными являются все функциональные параметры, за исключением $\phi(0, \xi), \psi(0, \xi)$ и $T(\xi)$. Процедуру решения этой системы нам удобно начать с уравнения (29). Перепишем его в виде

$$
d(\xi):=\Phi^{-1}(0, \mu) T^{-1}(\xi) \psi(0, \xi)=\tilde{\delta}_{u 2}(\xi) \delta_{u 2}^{-1}(\xi) .
$$

На основе явных выражений $(21),(22),(27)$ легко можно заключить, что левая часть последнего равенства нижнетреугольна, а правая - верхнетреугольна, следовательно, $d(\xi)$ - диагональная матрица, более того, $d(\xi) \equiv 1$. Полагая $\delta_{u 2}=1$, полностью исключим произвол и найдем, что $\tilde{\delta}_{u 2}=1$. Поэтому

$$
\Phi(0, \mu)=T^{-1}(\xi) \psi(0, \xi), \quad \xi \in I_{2} .
$$

Аналогичным образом решается уравнение (32). Его решение можно выбрать в виде

$$
\begin{aligned}
\Psi(0, \mu) & =T^{-1}(\xi) \phi(0, \xi), \quad \xi \in I_{5}, \\
\delta_{u 5}(\xi) & =\tilde{\delta}_{u 5}(\xi)=1 .
\end{aligned}
$$

Решение остальных уравнений системы (28)-(33) сводится к решению задачи о треугольной факторизации матрищ вида $\Phi^{-1}(0, \mu) T^{-1}(\xi) \phi(0, \xi)$ и $\Psi^{-1}(0, \mu) T^{-1}(\xi) \psi(0, \xi)$, поскольку функции $\Phi(0, \mu)$ и $\Psi(0, \mu)$ уже найдены. По найденным функциям $\Phi(0, \mu)$ и $\Psi(0, \mu)$ вычислим матрицу сопряжения (26) задачи Римана (25). В итоге поиск функций $\Phi(t, \mu)$ и $\Psi(t, \mu)$ при $t>0$ сведется к решению задачи Римана $(25),(26)$.

Найдем теперь эволюцию матрицы рассеяния во времени $t, t>0$. Выразим матрицу $s(\xi, t)$ в терминах решений задачи Римана (25), (26). Заметим прежде всего, что все матричные функции $s(\xi, t), \Phi(t, \mu)$ и $\Psi(t, \mu)$ удовлетворяют одному и тому же дифференциальному уравнению вида (23), причем две последние функции являются фундаментальными решениями этого уравнения. В силу того что столбцы $s_{1}(\xi, t)$ и $s_{2}(\xi, t)$ матрицы рассеяния определены в разных полуплоскостях, нам удобно рассмотреть их по отдельности. Например, $s_{1}(\xi, t)$ определен в полуплоскости $\operatorname{Im} \xi \geqslant 0$, т.е. в замыкании объединения секторов $I_{1}, I_{2}, I_{3}$. Легко показать, что имеют место следующие формулы разложения:

$$
\begin{aligned}
& s_{1}(\xi, t)=\Phi(t, \mu)\left(\begin{array}{c}
s_{11}(\xi, 0) \\
0
\end{array}\right), \quad \xi \in I_{2}, \\
& s_{1}(\xi, t)=\Psi(t, \mu)\left(\begin{array}{c}
s_{11}(\xi, 0)-r_{12}\left(\omega^{2} \xi\right) s_{21}(\xi, 0) \\
s_{21}(\xi, 0) e^{-8 i t \xi^{3}}
\end{array}\right), \quad \xi \in I_{1}, \\
& s_{1}(\xi, t)=\Psi(t, \mu)\left(\begin{array}{c}
s_{11}(\xi, 0)-r_{12}(\omega \xi) s_{21}(\xi, 0) \\
s_{21}(\xi, 0) e^{-8 i t \xi^{3}}
\end{array}\right), \quad \xi \in I_{3},
\end{aligned}
$$


где $\omega=e^{\frac{2 i \pi}{3}}-$ кубический корень из единицы, $r_{12}(\xi)=s_{12}(\xi, 0) s_{22}^{-1}(\xi, 0)$. Аналогично выражается второй столбец матрицы $s_{2}(\xi, t)$ :

$$
\begin{aligned}
& s_{2}(\xi, t)=\Psi(t, \mu)\left(\begin{array}{c}
0 \\
s_{22}(\xi, 0)
\end{array}\right), \quad \xi \in I_{5}, \\
& s_{2}(\xi, t)=\Phi(t, \mu)\left(\begin{array}{c}
s_{12}(\xi, 0) e^{8 i t \xi^{3}} \\
s_{22}(\xi, 0)-r_{21}(\omega \xi) s_{12}(\xi, 0)
\end{array}\right), \quad \xi \in I_{4}, \\
& s_{2}(\xi, t)=\Phi(t, \mu)\left(\begin{array}{c}
s_{12}(\xi, 0) e^{8 i t \xi^{3}} \\
s_{22}(\xi, 0)-r_{21}\left(\omega^{2} \xi\right) s_{12}(\xi, 0)
\end{array}\right), \quad \xi \in I_{6} .
\end{aligned}
$$

Здесь $r_{21}(\xi)=s_{21}(\xi, 0) s_{11}^{-1}(\xi, 0)$. Следуюший этап в решении начально-краевой задачи - воссстановление потенциала по заданной матрице рассеяния. Эта проблема хорошо изучена [18]. Она решается сведением либо к уравнениям Гельфанда-Левитана [18], либо к задаче Римана вида (15)-(19). О задаче Римана см., например, [20].

Благодарности. Работа поддержана Российским фондом фундаментальных исследований, грант № 98-01-00576.

\section{Список литературы}

[1] В.Е. Захаров, С.В. Манаков, С.П. Новиков, Л.П. Питаевский. Теория солитонов: Метод обратной задачи. М.: Наука, 1980.

[2] J. V. Moloney, A. C. Newell. Phys. Rev. A. 1989. V. 39. P. 1809.

[3] J. Bona, W. G. Pritchard, L. R. Scott. Philos. Trans. R. Soc. London A. 1981. V. 303. P. 457.

[4] J. Bona, R. Winther. SIAM J. Math. Anal. 1983. V. 14. P. 1056.

[5] C. K. Chu, R. L. Chou. Adv. Appl. Mech. 1990. V. 27. P. 283.

[6] A.S. Fokas. Physica D. 1989. V. 35. P. 167.

[7] А.С. Фокас, А.Р. Итс. ТМФ. 1992. Т. 92. № 3. С. 387.

[8] A.S. Fokas, A.R. Its. SIAM J. Math. Anal. 1996. V. 27. № 3. P. 738.

[9] M. J. Ablowitz, H. J. Segur. J. Math. Phys. 1975. V. 16. P. 1054.

[10] E. К. Склянин. Функц. анализ и его прилож. 1987. Т. 21. № 2. С. 86-87.

[11] V. O. Tarasov. Inverse Problems. 1991. V. 7. P. 435

[12] И. Т. Хабибуллин. ТМФ. 1991. Т. 86. № 1. С. 43.

[13] I. T. Habibullin. Phys. Lett. A. 1993. V. 178. P. 369.

[14] B. Gürel, M. Gürses, I. Habibullin. J. Math. Phys. 1995. V. 36. № 12. P. 6809.

[15] V. Adler, B. Gürel, M. Gürses, I. Habibullin. J. Phys. A. 1997. V. 30. P. 3505.

[16] В. Э. Адлер, И. Т. Хабибуллин, А. Б. Шабат. ТМФ. 1997. Т. 110. № 1. С. 98.

[17] М. Д. Рамазанов. Матем. сб. 1964. Т. 64. № 2. С. 234; An Ton Bui. J. Differ. Equ. 1977. V. 25. № 3. Р. 288; А. В. Фаминский. Труды ММО. 1988. Т. 51. С. 54.

[18] В. А. Марченко. Операторы Штурма-Лиувилля и их приложения. Киев: Наукова думка, 1977.

[19] А. Б. Шабат. Дифф. уравн. 1979. Т. 15. № 10. С. 1824.

[20] З. Пресдорф. Некоторые классы сингулярных уравнений. М.: Мир, 1979. 